\title{
An Optimal Feedforward Design for Complete PMD Compensation Up to the Second Order
}

\author{
George Ouyang, Avishay Eyal, Member, IEEE, and Amnon Yariv, Life Fellow, IEEE
}

\begin{abstract}
As an extension to a previous paper, this paper describes the optimization of a second-order, feedforward polarization-mode dispersion (PMD) compensation scheme by reducing its degrees of freedom (DOF) by two. The new design is optimal in the sense that the number of DOF used is the same as the minimal number of DOF required. Also derived is a set of constraint equations that govern the choice of various system parameters.
\end{abstract}

Index Terms-Compensation, differential group delay, feedforward systems, optical communication, polarization-mode dispersion (PMD).

\section{INTRODUCTION}

$\mathbf{S}$ NCE impairment due to polarization-mode dispersion (PMD) limits channel bit rate and transmission distance, PMD compensation is a key issue in high-speed, long-distance optical transmission. Various schemes, both feedback and feedforward, have been proposed to combat the PMD effect [1]-[5]. As a general rule, given an efficient scheme to extract the transmission fiber's PMD parameters, feedforward compensators are almost always the preferred method because they are faster and easier to implement, i.e., they do not require a feedback loop and a complex algorithm to optimize the control parameters.

In a previous paper [6], Phua et al. reported a feedforward technique capable of diagnosing and determining the relevant PMD parameters of a transmission fiber up to the second order. Based on the PMD information obtained using this technique, they presented in another paper [7] a compensation scheme using a deterministic approach. The resulting compensator, while straightforward in design, uses more degrees of freedom (DOF) than necessary. This paper serves to illustrate this point and tries to find ways to reduce the number of DOF to the most minimum value possible.

The paper is organized as follows. Section II reviews and summarizes the results of [7] and shows that the Phua compensator actually uses two DOF more than needed to fully compensate the PMD up to the second order. In Section III, solutions are proposed to reduce the extra DOF one by one.

Manuscript received July 24, 2003; revised April 29, 2004. This work was supported in part by the Office of Naval Research, by the Air Force Office of Scientific Research, and by the Defense Advanced Research Projects Agency (DARPA).

G. Ouyang and A. Yariv are with the California Institute of Technology, Pasadena, CA 91125 USA (e-mail: ouyang@caltech.edu).

A. Eyal is with Tel-Aviv University, Tel Aviv 69978, Israel.

Digital Object Identifier 10.1109/JLT.2004.832430

\section{REVIEW}

In Fig. 1, we redraw the compensator first proposed by Phua et al. in [7]. It consists of a series of alternating polarization rotators and frequency-independent delay segments.

$\mathrm{C}_{0}, \mathrm{C}_{1}, \mathrm{C}_{2}, \mathrm{R}_{1}, \mathrm{R}_{2}$, and $\mathrm{R}_{3}$ are the Muller rotation matrices. It is assumed that $\mathrm{C}_{0}, \mathrm{C}_{1}$, and $\mathrm{C}_{2}$ are frequency independent over the frequency range of interest, and $R_{1}, R_{2}$, and $R_{3}$ have negligible second-order and higher order PMD effects. Since the fiber parameters $\vec{\tau}_{f}$ and $\vec{\tau}_{\omega f}$ are known a priori [6], the only remaining issue is how to set the compensator appropriately so that the net PMD vectors $\vec{\tau}$ and $\vec{\tau}_{\omega}$ equal to zero.

From Fig. 1, the total first- and second-order PMD vectors $\vec{\tau}$ and $\vec{\tau}_{\omega}$ are found using the PMD vector concatenation rules [8]

$$
\begin{aligned}
\vec{\tau} & =\vec{\tau}_{c}+\mathrm{R}_{c} \mathrm{C}_{0} \vec{\tau}_{f} \\
\vec{\tau}_{\omega} & =\vec{\tau}_{\omega c}+\mathrm{R}_{c} \mathrm{C}_{0} \vec{\tau}_{\omega f}+\vec{\tau}_{c} \times \vec{\tau} .
\end{aligned}
$$

Setting $\vec{\tau}$ and $\vec{\tau}_{\omega}$ to zero as required for total PMD compensation, we find

$$
\begin{aligned}
\vec{\tau}_{c} & =-\mathrm{R}_{c} \mathrm{C}_{0} \vec{\tau}_{f} \\
\vec{\tau}_{\omega c} & =-\mathrm{R}_{c} \mathrm{C}_{0} \vec{\tau}_{\omega f} .
\end{aligned}
$$

Equations (3) and (4) clearly dictate that the vector pair $\left\{\vec{\tau}_{c}, \vec{\tau}_{\omega c}\right\}$ be related to $\left\{\vec{\tau}_{f}, \vec{\tau}_{\omega f}\right\}$ by a rigid body rotation. In other words, they impose the following requirements on $\vec{\tau}_{c}$ and $\vec{\tau}_{\omega c}:\left|\vec{\tau}_{c}\right|=\left|\vec{\tau}_{f}\right|,\left|\vec{\tau}_{\omega c}\right|=\left|\vec{\tau}_{\omega f}\right|$, and $\vec{\tau}_{c} \cdot \vec{\tau}_{\omega c}=\vec{\tau}_{f} \cdot \vec{\tau}_{\omega f}$. Constructing $\vec{\tau}_{c}$ and $\vec{\tau}_{\omega c}$ directly using Fig. 1 and the PMD vector concatenation rules, we arrive at a second set of expressions for $\vec{\tau}_{c}$ and $\vec{\tau}_{\omega c}$

$$
\begin{aligned}
\vec{\tau}_{c} & =\vec{\tau}_{3}+\mathrm{R}_{3} \mathrm{C}_{2} \vec{\tau}_{2}+\mathrm{R}_{3} \mathrm{C}_{2} \mathrm{R}_{2} \mathrm{C}_{1} \vec{\tau}_{1} \\
\vec{\tau}_{\omega c} & =\mathrm{R}_{3} \mathrm{C}_{2} \vec{\tau}_{2} \times \mathrm{R}_{3} \mathrm{C}_{2} \mathrm{R}_{2} \mathrm{C}_{1} \vec{\tau}_{1}+\left(\vec{\tau}_{3} \times \vec{\tau}_{c}\right) .
\end{aligned}
$$

Simplified, we get

$$
\begin{aligned}
& \overrightarrow{\mathrm{B}}+\overrightarrow{\mathrm{A}}=\vec{\tau}_{c}-\vec{\tau}_{3} \\
& \overrightarrow{\mathrm{B}} \times \overrightarrow{\mathrm{A}}=\vec{\tau}_{\omega c}-\left(\vec{\tau}_{3} \times \vec{\tau}_{c}\right)
\end{aligned}
$$

where

$$
\begin{aligned}
& \overrightarrow{\mathrm{B}}=\mathrm{R}_{3} \mathrm{C}_{2} \vec{\tau}_{2} \\
& \overrightarrow{\mathrm{A}}=\mathrm{R}_{3} \mathrm{C}_{2} \mathrm{R}_{2} \mathrm{C}_{1} \vec{\tau}_{1} .
\end{aligned}
$$

In [7], it is assumed that $\vec{\tau}_{2}$ and $\vec{\tau}_{3}$ are fixed and known PMD vectors, whereas $\vec{\tau}_{1}$ is fixed and known in orientation but adjustable in magnitude. Hence, from (9) and (10), it follows immediately that the vector $\overrightarrow{\mathrm{B}}$ is adjustable in orientation via rotation $\mathrm{C}_{2}$, while $\overrightarrow{\mathrm{A}}$ is adjustable in both magnitude and orientation 


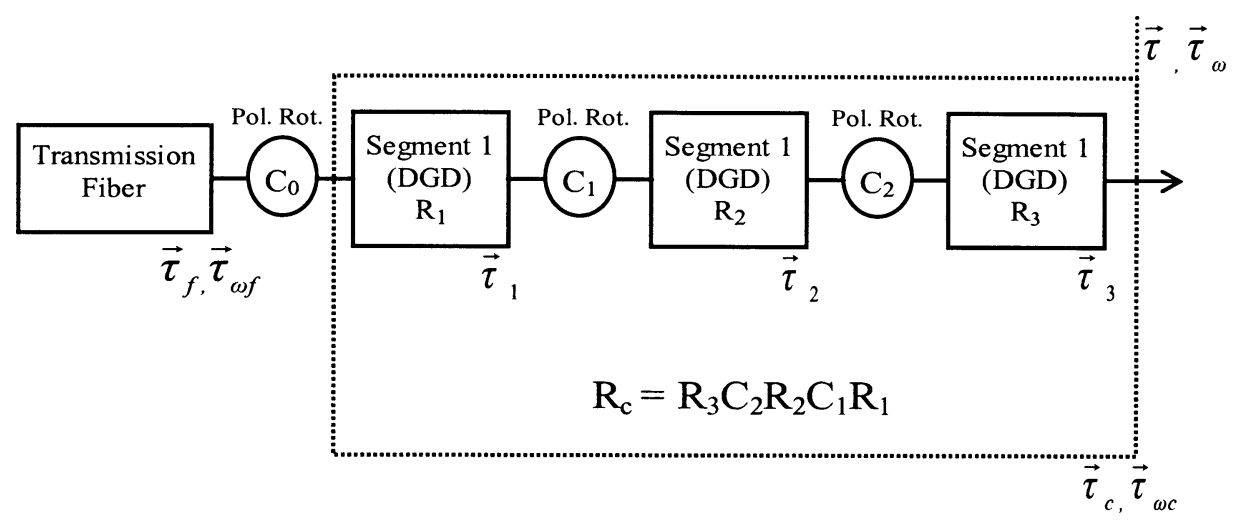

Fig. 1. Three-segment compensator proposed in [7]. It consists of three polarization rotators and three first-order PMD delay segments. Each segment gives a frequency-independent differential group delay (DGD), with $\vec{\tau}_{1}, \vec{\tau}_{2}$, and $\vec{\tau}_{3}$ being their respective PMD vectors. Note that in [7], the compensator refers only to the section in the dashed box whose first- and second-order PMD vectors are $\vec{\tau}_{c}$ and $\vec{\tau}_{\omega c}$, respectively, but in this paper, it is more convenient to also incorporate $\mathrm{C}_{0}$ into the compensator, as the DOF of the overall compensator system is to be considered.

via $\mathrm{C}_{1}$ and $\vec{\tau}_{1}$. The aim is to solve for $\overrightarrow{\mathrm{A}}$ and $\overrightarrow{\mathrm{B}}$ so that their respective rotation matrices $\mathrm{C}_{2}$ and $\mathrm{C}_{1}$ can be computed from (9) and (10).

First, we realize $\theta_{\omega 3}$, the angle between $\vec{\tau}_{\omega c}$ and $\vec{\tau}_{3}$, can be calculated from (7) and (8). Dot multiply the two equations, and we have

$$
\vec{\tau}_{\omega c} \cdot\left(\vec{\tau}_{c}-\vec{\tau}_{3}\right)=0
$$

Recall $\left|\vec{\tau}_{c}\right|=\left|\vec{\tau}_{f}\right|,\left|\vec{\tau}_{\omega c}\right|=\left|\vec{\tau}_{\omega f}\right|$, and $\vec{\tau}_{c} \cdot \vec{\tau}_{\omega c}=\vec{\tau}_{f} \cdot \vec{\tau}_{\omega f}$. We then find

$$
\cos \theta_{\omega 3}=\frac{\left|\vec{\tau}_{c}\right|}{\left|\vec{\tau}_{3}\right|} \cos \phi=\frac{\left|\vec{\tau}_{f}\right|}{\left|\vec{\tau}_{3}\right|} \cos \phi
$$

where $\phi$, the angle between $\vec{\tau}_{f}$ and $\vec{\tau}_{\omega f}$ (or between $\vec{\tau}_{c}$ and $\vec{\tau}_{\omega c}$ ), is determined a priori.

By requiring that $\vec{\tau}_{c}$ and $\vec{\tau}_{\omega c}$ lie on some arbitrary but conveniently chosen plane with $\vec{\tau}_{3}$ and knowing the angles $\theta_{\omega 3}$ and $\phi$, the positions of $\vec{\tau}_{c}$ and $\vec{\tau}_{\omega c}$ are also fixed. The vectors $\overrightarrow{\mathrm{A}}$ and $\overrightarrow{\mathrm{B}}$ can then be solved analytically. The solution of $\vec{B}$ is

$$
\overrightarrow{\mathrm{B}}=\left|\vec{\tau}_{2}\right|(\cos \psi \hat{\mathrm{p}}+\sin \psi \hat{\mathrm{q}})
$$

where $\hat{\mathrm{p}}$ is the unit vector in the direction of $\left(\vec{\tau}_{c}-\vec{\tau}_{3}\right)$, $\hat{\mathrm{q}}$ is the unit vector in the direction of $\left[\left(\vec{\tau}_{c}-\vec{\tau}_{3}\right) \times\left(\vec{\tau}_{\omega c}-\left(\vec{\tau}_{3} \times \vec{\tau}_{c}\right)\right)\right]$, and $\psi$ is the angle between $\overrightarrow{\mathrm{B}}$ and $\left(\vec{\tau}_{c}-\vec{\tau}_{3}\right)$ given by

$$
\sin \psi=\frac{\left|\vec{\tau}_{\omega c}-\left(\vec{\tau}_{3} \times \vec{\tau}_{c}\right)\right|}{\left|\vec{\tau}_{2}\right|\left|\vec{\tau}_{c}-\vec{\tau}_{3}\right|} .
$$

After solving for $\vec{B}, \vec{A}$ is solved using (7), where $\left|\vec{\tau}_{1}\right|$ is set to whatever value is needed to satisfy the equation. The rotation matrices $\mathrm{C}_{2}$ and $\mathrm{C}_{1}$ are then solved from (9) and (10)

$$
\begin{aligned}
& \mathrm{C}_{2} \vec{\tau}_{2}=\mathrm{R}_{3}^{+} \overrightarrow{\mathrm{B}} \\
& \mathrm{C}_{1} \vec{\tau}_{1}=\left(\mathrm{R}_{3} \mathrm{C}_{2} \mathrm{R}_{2}\right)+\overrightarrow{\mathrm{A}} .
\end{aligned}
$$

Knowing $\mathrm{C}_{1}$ and $\mathrm{C}_{2}, \mathrm{C}_{0}$ follows from (3) and (4)

$$
\begin{aligned}
\mathrm{C}_{0} \vec{\tau}_{f} & =-\left(\mathrm{R}_{c}\right)^{+} \vec{\tau}_{c} \\
\mathrm{C}_{0} \vec{\tau}_{\omega f} & =-\left(\mathrm{R}_{c}\right)^{+} \vec{\tau}_{\omega c} .
\end{aligned}
$$

The total number of DOF of the system can be computed from (15)-(18). It is well known that two DOF are required to transform a fixed polarization direction (e.g., $\left.\vec{\tau}_{2}\right)$ to any arbitrary direction (e.g., $R_{3}^{+} \vec{B}$ ) and three DOF to transform a pair of fixed polarizations (e.g., $\left.\left\{\vec{\tau}_{f}, \vec{\tau}_{\omega f}\right\}\right)$ through a rigid body rotation to a pair of arbitrary polarizations (e.g., $\left\{\vec{\tau}_{c}, \vec{\tau}_{\omega c}\right\}$ ). This means that $\mathrm{C}_{1}$ and $\mathrm{C}_{2}$ each contain two DOF while $\mathrm{C}_{0}$ contains three. In addition, there is one more DOF contributed by the variable delay $\left|\vec{\tau}_{1}\right|$. This brings the total number of DOF of the system to

Total number of DOF of the system $=2+2+3+1=8$.

However, the minimal number of DOF needed for complete PMD compensation is only six (three for $\vec{\tau}_{f}$ and three for $\vec{\tau}_{\omega f}$ ). This means that the Phua compensator contains two DOF too many, and in theory, we should be able to remove the redundancy with a better design. Furthermore, it is worth noting that the system also comes with two constraint equations, namely (12) and (14). Both equations have to have solutions in order to ensure robust PMD compensation, which in turn imposes constraints upon the magnitudes of $\vec{\tau}_{2}$ and $\vec{\tau}_{3}$ [7].

\section{DESIGN ModifiCATIONS}

\section{A. Fix the Magnitude of $\vec{\tau}_{1}$}

The compensator system we propose has the same structure as that in [7] (also see Fig. 1). The only difference is that the magnitude of $\vec{\tau}_{1}$ is now fixed to reduce the total number of DOF by one. This change in design has practical significance as well, for a fixed differential-group-delay (DGD) segment introduces a smaller loss than a variable-delay segment and is less likely to require an optical amplifier [7].

Equations (1)-(12) are still valid, for the structure of the compensator remains unchanged. Specifically, this means the angle between $\vec{\tau}_{c}$ and $\vec{\tau}_{\omega c}$ is still $\phi$ and the one between $\vec{\tau}_{\omega c}$ and $\vec{\tau}_{3}$ still $\theta_{\omega 3}$. We do not, however, require in our new scheme that $\vec{\tau}_{3}, \vec{\tau}_{c}$, and $\vec{\tau}_{\omega c}$ be coplanar. Instead, we set the direction of $\vec{\tau}_{3}$ to be the $z$ direction and the plane spanned by $\vec{\tau}_{3}$ and $\vec{\tau}_{\omega c}$ the $y z$ plane. $\vec{\tau}_{c}$ is then located on a cone centered around $\vec{\tau}_{\omega c}$, as shown in Fig. 2. 


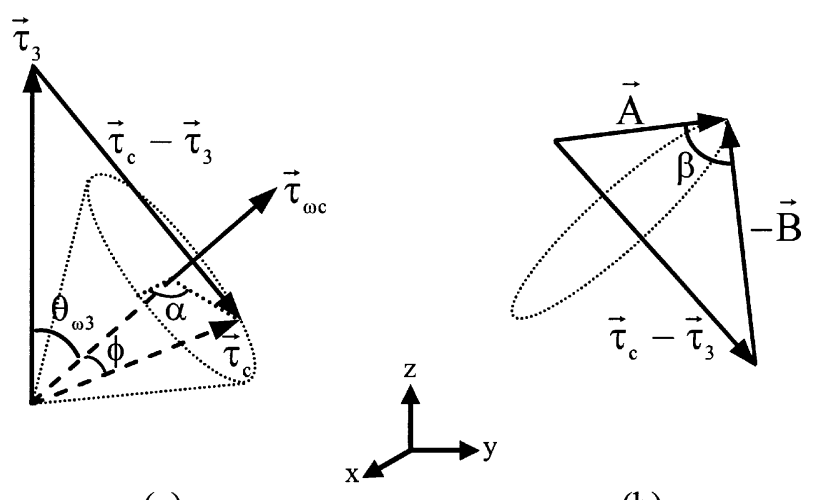

(a)

(b)

Fig. 2. Relative orientations of $\vec{\tau}_{3}, \vec{\tau}_{c}, \vec{\tau}_{\omega c}, \overrightarrow{\mathrm{A}}$, and $\overrightarrow{\mathrm{B}}$. (a) $\vec{\tau}_{3}$ and $\vec{\tau}_{\omega c}$ lie in the $y z$ plane, and $\theta_{\omega 3}$ is the angle between them. $\vec{\tau}_{c}$ lies on a cone centered on $\vec{\tau}_{\omega c}$ with an apex angle $\phi$. The orientation of $\vec{\tau}_{c}$ is determined by $\alpha$, the azimuthal angle on the bottom surface of the cone. (b) $\vec{A},-\vec{B}$, and $\left(\vec{\tau}_{c}-\vec{\tau}_{3}\right)$ form a triangle, and $\beta$ is the angle between $\vec{A}$ and $-\vec{B}$. Note the triangle is free to rotate about $\left(\vec{\tau}_{c}-\vec{\tau}_{3}\right)$ to whatever orientation needed to satisfy (8).

In vectorial form

$$
\vec{\tau}_{3}=\left|\vec{\tau}_{3}\right|\left(\begin{array}{l}
0 \\
0 \\
1
\end{array}\right), \quad \vec{\tau}_{\omega c}=\left|\vec{\tau}_{\omega c}\right|\left(\begin{array}{c}
0 \\
\sin \theta_{\omega c} \\
\cos \theta_{\omega c}
\end{array}\right)
$$

and

$$
\vec{\tau}_{c}=\left|\vec{\tau}_{c}\right|\left(\begin{array}{c}
\sin \phi \cos \alpha \\
\cos \theta_{\omega c} \sin \phi \sin \alpha+\sin \theta_{\omega c} \cos \phi \\
-\sin \theta_{\omega c} \sin \phi \sin \alpha+\cos \theta_{\omega c} \cos \phi
\end{array}\right) .
$$

The direction of $\vec{\tau}_{c}$ is given by the azimuthal angle $\alpha$, which is yet to be determined at this point. To solve for $\alpha$, we go back to (7) and (8). Although both equations are vectorial in nature, we claim that it is only the magnitude we have to worry about. For (7), we recall both $\vec{A}$ and $\vec{B}$ are adjustable in orientation. Therefore, provided that $|\overrightarrow{\mathrm{A}}|$ and $|\overrightarrow{\mathrm{B}}|$ are sufficiently large, they will always be able to form a triangle with $\left(\vec{\tau}_{c}-\vec{\tau}_{3}\right)$ as in Fig. 2(b), hence, satisfying (7). For (8), we notice all three terms involved are perpendicular to $\left(\vec{\tau}_{c}-\vec{\tau}_{3}\right)$ (see (7) and (11)), or in other words, $(\overrightarrow{\mathrm{B}} \times \overrightarrow{\mathrm{A}}), \vec{\tau}_{\omega c}$, and $\left(\vec{\tau}_{3} \times \vec{\tau}_{c}\right)$ are all located in a plane perpendicular to $\left(\vec{\tau}_{c}-\vec{\tau}_{3}\right)$. Moreover, because $\vec{A}$ and $\vec{B}$ are adjustable in orientation, we can rotate $(\vec{B} \times \vec{A})$ to point in any direction in this plane [by rotating the triangle around $\left(\vec{\tau}_{c}-\vec{\tau}_{3}\right)$ in Fig. 2(b)] without changing its magnitude. Hence, (8) will also be satisfied as long as they are satisfied in magnitude.

We proceed by taking the squared magnitude on both sides of (7) and (8), as follows:

$$
\begin{aligned}
& |\overrightarrow{\mathrm{B}}+\overrightarrow{\mathrm{A}}|^{2}=|\overrightarrow{\mathrm{A}}|^{2}+|\overrightarrow{\mathrm{B}}|^{2}-2|\overrightarrow{\mathrm{A}}||\overrightarrow{\mathrm{B}}| \cos \beta=\left|\vec{\tau}_{c}-\vec{\tau}_{3}\right|^{2} \\
& |\overrightarrow{\mathrm{B}} \times \overrightarrow{\mathrm{A}}|^{2}=|\overrightarrow{\mathrm{A}}|^{2}|\overrightarrow{\mathrm{B}}|^{2} \sin ^{2} \beta=\left|\vec{\tau}_{\omega c}-\left(\vec{\tau}_{3} \times \vec{\tau}_{c}\right)\right|^{2}
\end{aligned}
$$

where $\beta$ is the angle between $\vec{A}$ and $\vec{B}$. However, $|\overrightarrow{\mathrm{A}}|^{2}|\overrightarrow{\mathrm{B}}|^{2} \sin ^{2} \beta$ in (22) is also given by the trigonometric identity

$$
|\overrightarrow{\mathrm{A}}|^{2}|\overrightarrow{\mathrm{B}}|^{2} \sin ^{2} \beta=|\overrightarrow{\mathrm{A}}|^{2}|\overrightarrow{\mathrm{B}}|^{2}-|\overrightarrow{\mathrm{A}}|^{2}|\overrightarrow{\mathrm{B}}|^{2} \cos ^{2} \beta .
$$

Solve for $|\overrightarrow{\mathrm{A}}||\overrightarrow{\mathrm{B}}| \cos \beta$ using (21) and substitute it into (23), and we have

$$
|\overrightarrow{\mathrm{A}}|^{2}|\overrightarrow{\mathrm{B}}|^{2} \sin ^{2} \beta=|\overrightarrow{\mathrm{A}}|^{2}|\overrightarrow{\mathrm{B}}|^{2}-\frac{1}{4}\left[|\overrightarrow{\mathrm{A}}|^{2}+|\overrightarrow{\mathrm{B}}|^{2}-\left|\vec{\tau}_{c}-\vec{\tau}_{3}\right|^{2}\right]^{2} \text {. }
$$

Equate the right sides of (22) and (24), and we get

$$
\left|\vec{\tau}_{\omega c}-\left(\vec{\tau}_{3} \times \vec{\tau}_{c}\right)\right|^{2}=|\overrightarrow{\mathrm{A}}|^{2}|\overrightarrow{\mathrm{B}}|^{2}-\frac{1}{4}\left[|\overrightarrow{\mathrm{A}}|^{2}+|\overrightarrow{\mathrm{B}}|^{2}-\left|\vec{\tau}_{c}-\vec{\tau}_{3}\right|^{2}\right]_{(25)}^{2} \text {. }
$$

Substituting into (19) and (20) and noting $|\overrightarrow{\mathrm{A}}|=\left|\vec{\tau}_{1}\right|,|\overrightarrow{\mathrm{B}}|=$ $\left|\vec{\tau}_{2}\right|,\left|\vec{\tau}_{c}\right|=\left|\vec{\tau}_{f}\right|$, and $\left|\vec{\tau}_{\omega c}\right|=\left|\vec{\tau}_{\omega f}\right|$, (25) becomes (26), shown at the bottom of the page, which can be transformed into a quadratic equation in $\cos \alpha$ (or $\sin \alpha$ ). Thus, $\alpha$ can be solved from (26), and $\vec{\tau}_{c}$ and $\beta$ can be determined from (20) and (21), respectively. Once $\vec{\tau}_{c}$ and $\beta$ are known, the orientations of $\vec{A}$ and $\overrightarrow{\mathrm{B}}$ can be easily solved from (7) and (8).

We note that (12), (21), and (25) now serve as the constraint equations of the new system. To ensure robust PMD compensation, we need to choose the magnitude of $\vec{\tau}_{1}, \vec{\tau}_{2}$, and $\vec{\tau}_{3}$ appropriately so that we can always have solutions for the three constraint equations with any anticipated magnitude of $\vec{\tau}_{f}$ and $\vec{\tau}_{\omega f}$.

It is worth comparing the new set of constraint equations with those of Phua's original design [7], in which (12) and (14) provide the constraint conditions. In the new scheme, we kept (12), got rid of (14), and added (21) and (25). Equation (21) in principle does not impose too severe a constraint on the system. For instance, if we set

$$
|\overrightarrow{\mathrm{A}}|=|\overrightarrow{\mathrm{B}}| \geq \frac{1}{2} \operatorname{Max}\left|\vec{\tau}_{c}-\vec{\tau}_{3}\right|
$$

then

$$
\operatorname{Min}|\overrightarrow{\mathrm{A}}+\overrightarrow{\mathrm{B}}|=0, \quad \operatorname{Max}|\overrightarrow{\mathrm{A}}+\overrightarrow{\mathrm{B}}| \geq \operatorname{Max}\left|\vec{\tau}_{c}-\vec{\tau}_{3}\right|
$$

or, equivalently

$$
0=\operatorname{Min}|\overrightarrow{\mathrm{A}}+\overrightarrow{\mathrm{B}}| \leq\left|\vec{\tau}_{c}-\vec{\tau}_{3}\right| \leq \operatorname{Max}|\overrightarrow{\mathrm{A}}+\overrightarrow{\mathrm{B}}|
$$

and (21) is satisfied for all time (i.e., a $\beta$ angle can always be found). That leaves us with (25), which basically replaces (14) in the original design as the more restrictive condition that could potentially cause problems in actual implementation. Neither of these two conditions is straightforward to implement, and their satisfaction requires further numerical and experimental analysis.

$$
\begin{aligned}
& 2\left|\vec{\tau}_{f} \| \vec{\tau}_{\omega f}\right|\left|\vec{\tau}_{3}\right| \sin \theta \sin \phi \cos \alpha+\left|\vec{\tau}_{f}\right|\left|\vec{\tau}_{3}\right|\left[\left|\vec{\tau}_{1}\right|^{2}+\left|\vec{\tau}_{2}\right|^{2}-\left|\vec{\tau}_{3}\right|^{2}-\left|\vec{\tau}_{f}\right|^{2}\right] \sin \theta \sin \phi \sin \alpha \\
& \quad=\left|\vec{\tau}_{f}\right|^{2}\left|\vec{\tau}_{3}\right|^{2}+\left|\vec{\tau}_{\omega f}\right|^{2}-\left|\vec{\tau}_{1}\right|^{2}\left|\vec{\tau}_{2}\right|^{2}+\frac{1}{4}\left[\left|\vec{\tau}_{1}\right|^{2}+\left|\vec{\tau}_{2}\right|^{2}-\left|\vec{\tau}_{f}\right|^{2}-\left|\vec{\tau}_{3}\right|^{2}\right]^{2}+\left|\vec{\tau}_{f}\right|\left|\vec{\tau}_{3}\right|\left[\left|\vec{\tau}_{1}\right|^{2}+\left|\vec{\tau}_{2}\right|^{2}-\left|\vec{\tau}_{f}\right|^{2}-\left|\vec{\tau}_{3}\right|^{2}\right] \cos \theta \cos \phi
\end{aligned}
$$




\section{B. Reduce the Number of DOF in $\mathrm{C}_{0}$ by One}

In both designs discussed so far, the polarization rotator $\mathrm{C}_{0}$ contains three DOF and is required to perform a rigid body rotation between two pairs of time-varying states of polarization (SOP) (see (17) and (18)). We can represent this transformation in the Jones space by a generic SU(2) matrix

$$
\mathrm{C}_{0}=\left(\begin{array}{cc}
\cos \varphi \exp (\mathrm{i} \gamma) & \sin \varphi \exp (\mathrm{i} \eta) \\
-\sin \varphi \exp (-\mathrm{i} \eta) & \cos \varphi \exp (-\mathrm{i} \gamma)
\end{array}\right)
$$

where the angles $\varphi, \gamma$, and $\eta$ are determined by solving (17) and (18).

It has long been demonstrated that wave-plate polarization rotators are capable of continuously transforming a time-varying SOP of arbitrary polarization into a time-varying SOP of another polarization [9]. However, such devices contain only two DOF and work on only one SOP at a time. We can, of course, always use two such rotators to implement $\mathrm{C}_{0}$, but we show in this section that with a slight modification in our scheme, we can reduce the number of DOF in $\mathrm{C}_{0}$ by one, hence making it realizable with a single polarization rotator. (We would like to point out that for continuous and reset-free operations, all commercially available polarization rotators today require three DOF. The third DOF, however, is employed purely for engineering reasons and should be differentiated from the first two "essential" DOF [10].)

In the last section, we begin with the assumption that $\vec{\tau}_{3}$ and $\vec{\tau}_{\omega c}$ are in the $y z$ plane and then proceed to find $\overrightarrow{\mathrm{A}}$ and $\overrightarrow{\mathrm{B}}$. After a solution for $\vec{A}$ and $\vec{B}$ has been found, or in other words, after we have constructed a configuration of vectors in Fig. 2 that satisfies (7) and (8), we realize that it remains a solution even if we rotate all the vectors around $\vec{\tau}_{3}$ by a fixed but arbitrary angle $\delta$. This amounts to redefining every vector in (7) and (8) (and in Fig. 2) by applying on them a rigid body rotation, an operator that conserves equalities (7) and (8).

A rotation about the $z$ axis by a fixed angle $\delta$ in the Stokes space gets translated into the Jones space as [8]

$$
\mathrm{R}_{\mathrm{z}}\left(\begin{array}{cc}
\exp \left(-\frac{\mathrm{i} \delta}{2}\right) & 0 \\
0 & \exp \left(\frac{\mathrm{i} \delta}{2}\right)
\end{array}\right) \text {. }
$$

Apply the above rotation operator after the original $\mathrm{C}_{0}$ matrix, and we get

$$
\begin{aligned}
\mathrm{C}_{0}^{(\text {new })} & \\
& =\mathrm{R}_{\mathrm{z}} \mathrm{C}_{0} \\
& =\left(\begin{array}{cc}
\cos \alpha \exp \left[\mathrm{i}\left(\gamma-\frac{\delta}{2}\right)\right] & \sin \alpha \exp \left[\mathrm{i}\left(\eta-\frac{\delta}{2}\right)\right] \\
-\sin \alpha \exp \left[-\mathrm{i}\left(\eta-\frac{\delta}{2}\right)\right] & \cos \alpha \exp \left[-\mathrm{i}\left(\gamma-\frac{\delta}{2}\right)\right]
\end{array}\right) .
\end{aligned}
$$

Choose $\delta=\gamma+\eta$ and define $\Omega=(\gamma-\eta) / 2$, and $\mathrm{C}_{0}^{(\text {new })}$ becomes

$$
\mathrm{C}_{0}^{(\text {new })}=\left(\begin{array}{cc}
\cos \alpha \exp (\mathrm{i} \Omega) & \sin \alpha \exp (-\mathrm{i} \Omega) \\
-\sin \alpha \exp (\mathrm{i} \Omega) & \cos \alpha \exp (-\mathrm{i} \Omega)
\end{array}\right)
$$

and we obtain a rotation matrix with only two DOF.

\section{CONCLUSION}

In this paper, a feedforward PMD compensator capable of complete PMD compensation up to the second order is optimized. A reduction in cost and complexity by minimizing the total number of DOF of the system is achieved. Also derived is a new set of constraint equations, which govern the choice of various system parameters. More analytic and simulation work is required to compare the performance of the two designs as well as to verify the validity of our approach in general. This will be the subject of a future publication.

\section{ACKNOWLEDGMENT}

The authors would like to thank Y. Park of the Office of Naval Research, H. Schlossberg of the Air Force Office of Scientific Research, and R. Athale of DARPA for their support. G. Ouyang would like to thank Dr. X. Wu for sound suggestions and fruitful discussions.

\section{REFERENCES}

[1] H. Y. Pua, K. Peddanarappagari, B. Zhu, C. Allen, K. Demarest, and R. Hui, "An adaptive first-order polarization-mode dispersion compensation system aided by polarization scrambling: Theory and demonstration," J. Lightwave Technol., vol. 18, pp. 832-840, June 2000.

[2] P. C. Chou, J. M. Fini, and H. A. Haus, "Demonstration of a feed-forward PMD compensation technique," IEEE Photon. Technol. Lett., vol. 14, pp. 161-163, Feb. 2002

[3] H. Sunnerud, C. Xie, M. Karlsson, R. Samuelsson, and P. A. Andrekson, "A comparison between different PMD compensation techniques," $J$. Lightwave Technol., vol. 20, pp. 368-378, Mar. 2002.

[4] L.-S. Yan, Q. Yu, A. B. Sahin, and A. E. Willner, "Differential group delay monitoring used as feedforward information for polarization mode dispersion compensation," IEEE Photon. Technol. Lett., vol. 14 pp. 1463-1465, Oct. 2002.

[5] Z. Pan, Y. Wang, C. Yu, T. Luo, A. B. Sahin, Q. Yu, and A. E. Willner, "Intrabit polarization diversity modulation for the mitigation of PMD effects," IEEE Photon. Technol. Lett., vol. 14, pp. 1466-1468, Oct. 2002.

[6] P. B. Phua, J. M. Fini, and H. A. Haus, "Real-time first and second order PMD characterization using averaged state-of-polarization of filtered signal and polarization scrambling," J. Lightwave Technol., vol. 21 , pp. 982-989, Apr. 2003

[7] P. B. Phua and H. A. Haus, "Deterministic approach to first- and secondorder PMD compensation," IEEE Photon. Technol. Lett., vol. 14, pp. 1270-1272, Sept. 2002.

[8] J. P. Gordon and H. Kogelnik, "PMD fundamentals: Polarization mode dispersion in optical fibers," in Proc. Nat. Academy Science, vol. 97, Apr. 25, 2000, pp. 4541-4550.

[9] N. G. Walker and G. R. Walker, "Polarization control for coherent communications," J. Lightwave Technol., vol. 8, pp. 438-458, Mar. 1990.

[10] F. Heismann, "Analysis of a reset-free polarization controller for fast automatic polarization stabilization in fiber-optic transmission systems," J. Lightwave Technol., vol. 12, pp. 690-699, Apr. 1994.

George Ouyang, photograph and biography not available at the time of publication.

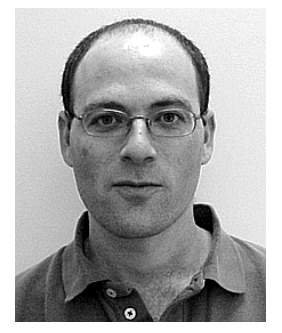

Avishay Eyal (S'95-M'99) received the B.Sc. degree in electrical engineering from The TechnionIsrael Institute of Technology, Haifa, Israel, in 1989, the M.S. degree from Ben-Gurion University, Beer-Sheva, Israel, in 1993, and the Ph.D. degree from Tel-Aviv University, Tel Aviv, Israel, in 1999 , with a dissertation that focused on the theoretical and experimental study of polarization-related effects in fiber-optic systems and lasers.

He became a Postdoctoral Fellow at the California Institute of Technology (CALTECH), Pasadena, in 1999. His research at CALTECH focused on modeling of light propagation in optical fibers and studying approaches for mitigation of propagation-related signal distortions. In 2002 , he returned to the engineering faculty at Tel-Aviv University, where he is currently teaching and conducting research in the area of optical communications.

Amnon Yariv (S'56-M'59-F'70-LF'95), photograph and biography not available at the time of publication. 